\title{
Loss of hepatocyte identity following aberrant YAP activation: a key mechanism in alcoholic hepatitis
}

Authors: Mohamed Bou Saleh ${ }^{1}$, Alexandre Louvet ${ }^{1}$, Line Carolle Ntandja-Wandji ${ }^{1}$, Emmanuel Boleslawski ${ }^{2}$, Viviane Gnemmi ${ }^{3}$, Guillaume Lassailly ${ }^{1}$, Stéphanie Truant ${ }^{4}$, François Maggiotto $^{1}$, Massih Ningarhari ${ }^{1}$, Florent Artru ${ }^{1}$, Emilie Anglo ${ }^{1}$, Pau Sancho-Bru $^{5}$, Anne Corlu ${ }^{6}$, Josepmaria Argemi ${ }^{7}$, Julie Dubois-Chevalier ${ }^{8}$, Sébastien Dharancy ${ }^{1}$, Jérôme Eeckhoute ${ }^{8}$, Ramon Bataller ${ }^{7}$, Philippe Mathurin ${ }^{1 *}$, Laurent Dubuquoy ${ }^{1 *}$.

\section{Affiliations:}

${ }^{1}$ Univ. Lille, Inserm, CHU Lille, U1286 - INFINITE - Institute for Translational Research in Inflammation, F-59000 Lille, France

${ }^{2}$ Univ. Lille, Inserm, CHU Lille, U1189 - ONCO-THAI - Image Assisted Laser Therapy for Oncology, F-59000 Lille, France

${ }^{3}$ CHU Lille, Service d'Anatomopathologie, F-59000 Lille, France.

${ }^{4}$ CHU Lille, Service de Chirurgie Digestive et Transplantations, F-59000 Lille, France.

5 Institut d'Investigacions Biomèdiques August Pi i Sunyer (IDIBAPS), University of Barcelona, Centro de Investigación Biomédica en Red de Enfermedades Hepáticas y Digestivas (CIBERehd), Barcelona, Spain.

${ }^{6}$ INSERM, Univ Rennes, INRAE, Institut NuMeCan (Nutrition, Metabolisms and Cancer), F35043, Rennes, France.

${ }^{7}$ Division of Gastroenterology, Hepatology and Nutrition. Pittsburgh Liver Research Center. University of Pittsburgh Medical Center (UPMC), Pittsburgh, PA, USA.

${ }^{8}$ Univ. Lille, Inserm, CHU Lille, Institut Pasteur de Lille, U1011-EGID, F-59000 Lille, France 


\section{*To whom correspondence should be addressed:}

- Laurent Dubuquoy PhD, Infinite - U1286, 4e étage Est, Faculté de Médecine - Pôle Recherche, 1 Place Verdun, F-59045 Lille CEDEX, France. E-mail: laurent.dubuquoy@inserm.fr; phone: +33-320-974-208, fax: +33-320-623-525.

- Philippe Mathurin MD, PhD, Service des Maladies de l'Appareil Digestif et de la Nutrition, Hôpital Claude Huriez, rue Michel Polonovski, F-59037 Lille CEDEX, France. E-mail: philippe.mathurin@chru-lille.fr; phone: +33-320-445-597.

Key words: hepatocyte, Hippo/YAP, transdifferentiation, alcoholic hepatitis, regeneration

Electronic word count: 6064 words excluding title page

\section{Number of figures and tables: 7}

Conflict of interest statements: The authors declare that they have no competing interests in relation to this manuscript.

Financial support statement: The authors acknowledge funding from the National Institute on Alcohol Abuse and Alcoholism (NIAAA/NIH; grant 1U01AA021908), Association Française pour l'Etude du Foie (AFEF), Institut national de la santé et de la recherche médicale (INSERM), CHU de Lille and Région Haut de France. Work at INSERM U1011 was supported by grants from the Fondation pour la Recherche Médicale (Equipe labellisée, DEQ20150331724), “European Genomic Institute for Diabetes” (E.G.I.D., ANR-10-LABX46) and European Commission. PS-B was supported by Fondo de Investigación Sanitaria Carlos III (FIS), co-financed by Fondo Europeo de Desarrollo Regional (FEDER), Unión Europea, "Una manera de hacer Europa" (FIS PI17/00673, and from the NIAAA grant 1U01AA021908-01-33490.

Author contributions: MBS performed research and participated in study and experiments design, contributed to the acquisition, analysis and interpretation of data. FM, VG, GL, LCNW, MN, FA, EA, PSB, AC, JA, JDC, JE, AL, PM and LD contributed to the acquisition, analysis 
or interpretation of data. $\mathrm{AL}, \mathrm{SD}$, and $\mathrm{PM}$ designed the clinical cohorts and supervised enrolment. EB, ST, MBS and LD supervised the specimen collection. LD conceived and designed the overall study. PM, RB and LD provided the financial support. MBS, PM and LD prepared the manuscript; all authors reviewed and edited the manuscript.

Institutional review board reference: CPP 2014-A01452-45 and DC-2008-642

Data availability statement: The RNA-seq raw data (Fig. 1A) have been deposited in the Database of Genotypes and Phenotypes (dbGAP) of the National Center for Biotechnology Information (United States National Library of Medicine, Bethesda, MD) under accession number phs001807.v1.p1. Microdissected liver RNAseq data (Fig. 3, Fig.S6) are available in NCBI's Gene Expression Omnibus repository as GSE167308. 


\section{ABSTRACT:}

Background \& Aims: Alcoholic hepatitis (AH) is a life-threatening disease with limited therapeutic options, because understanding of the molecular drivers leading to death are not well understood. This study evaluates the Hippo/Yes-associated protein (YAP) pathway which has been shown to play a role in liver regeneration. Method: The Hippo/YAP pathway was dissected in explants of patients transplanted for $\mathrm{AH}$ or alcoholic cirrhosis and in control livers, using RNA-Seq, real-time PCR, Western blot, immunohistochemistry (IHC) and transcriptome analysis after laser microdissection. We transfected primary human hepatocytes with constitutively active YAP (YAPS127A) and treated HepaRG cells and primary hepatocytes isolated from AH livers with a YAP inhibitor. We also used mouse models of ethanol exposure (Lieber de Carli) and liver regeneration $\left(\mathrm{CCl}_{4}\right)$ after hepatocyte transduction of YAPS127A. Results: In AH samples RNA-Seq analysis and IHC of total liver and microdissected hepatocytes revealed marked down-regulation of Hippo shown by lower MST1 kinase and abnormal activation of YAP in hepatocytes. Overactivation of YAP in hepatocytes in vitro and in vivo led to biliary differentiation and loss of key biological functions such as regeneration capacity. Conversely, treatment of abnormal hepatocytes from $\mathrm{AH}$ patients with a YAP inhibitor restored the mature hepatocyte phenotype. In ethanol-fed mice, YAP activation using YAPS127A resulted in a loss of hepatocyte differentiation. Hepatocyte proliferation was hampered using YAPS127A after $\mathrm{CCl}_{4}$ intoxication. Conclusion: Aberrant activation of YAP plays an important role in hepatocyte transdifferentiation in $\mathrm{AH}$, through a loss of hepatocyte identity and impaired regeneration. Thus, targeting YAP is a promising strategy for the treatment of patients with $\mathrm{AH}$. 
Lay summary: Alcoholic hepatitis $(\mathrm{AH})$ is characterized by inflammation and a lifethreatening alteration of liver regeneration by mechanisms that have not been identified. We show that AH livers are characterized by profound deregulation of the Hippo/YAP pathway with uncontrolled activation of the transcription co-factor YAP in hepatocytes. YAP activation in hepatocytes leads to their transdifferentiation towards a biliary phenotype associated with inflammation as well as a regeneration defect. YAP inhibition reverts this hepatocyte defect and appears to be an original therapeutic strategy of regenerative treatment for $\mathrm{AH}$. 


\section{INTRODUCTION}

Alcohol-associated liver disease (ALD) is a major public health problem worldwide. Alcoholic hepatitis (AH) is the most life-threatening phenotype, and effective targeted therapies are urgently needed. Severe forms of $\mathrm{AH}$ have a high short-term mortality rate - and the only effective therapy (i.e. corticosteroids) was proposed several decades ago [1] [2]. Although corticosteroids increase short-term survival [3], around $40 \%$ of patients fail to respond to treatment [4]. In a carefully selected group of patients, early liver transplantation is associated with a good outcome, but can only be proposed in a limited number of patients [5]. Since the only existing effective treatment is corticosteroids, new molecular pathways and targets must be identified.

Cell injury, inflammation, bacterial compounds, oxidative stress and regeneration are key factors of alcohol-induced liver injury [6] [7] [8]. Preclinical animal models do not mimic the full spectrum of severe $\mathrm{AH}$. Indeed, liver injury is mild in animals while profound impairment of liver function is a major feature of $\mathrm{AH}$ in humans. Thus, studies combining animal models, experimental cells and human tissue samples are needed to overcome the limitations of each individual approach [9].

In-depth studies are hampered in humans by the limited access to liver biopsies and thus, to RNA and histological analyses. However, indications for early liver transplantation in patients with severe $\mathrm{AH}$ have opened up a new era and facilitated molecular investigations, by making large amounts of explanted liver tissue available [5]. Recent studies of explants have revealed that ineffective hepatocyte regeneration and compensatory ductular reaction are key features of $\mathrm{AH}$ [10] [11] [12]. The molecular mechanisms underlying hepatocyte-transformation into biliary cells or so-called transdifferentiation, have been studied in mice but have not been explored in human diseases including AH. Identification of key drivers could help the 
development of novel therapeutic strategies to promote effective hepatocyte regeneration by shifting transdifferentiated cells towards a mature hepatocyte phenotype.

The Hippo/Yes-associated protein (YAP) pathway is a promising therapeutic target involved in liver regeneration [13]. This pathway has mainly been studied in animal models. Inactivation of the Hippo pathway leads to translocation of the transcription cofactor YAP into the nucleus, inducing transcription of a broad range of genes [14]. Although YAP is mainly expressed in biliary cells in the healthy liver, published preclinical data suggest that modulation of the Hippo/YAP pathway could influence embryonic liver development [15], cell fate [16], and liver regeneration [14]. Indeed, in mice, ectopic YAP activation in hepatocytes promotes transdifferentiation to an intermediate phenotype expressing both hepatocyte and biliary cell markers [16] and a ductular reaction [17], a histological feature frequently observed in $\mathrm{AH}$ [12] [10]. YAP activation is also associated with an increase in liver size [18] [19]. The relevance of all these results in animals must be determined in human pathophysiology.

In the present human-explant-based translational study, we investigated the role of the Hippo/YAP pathway in severe AH, a disease characterized by hepatocyte transdifferentiation into cholangiocytes as well as a lack of proliferating hepatocytes. In addition to our ex vivo data we also obtained functional in vivo data on the dynamics of YAP activation. Our results show that (i) deregulation of the Hippo/YAP pathway is closely associated with hepatocellular and regeneration defects observed in $\mathrm{AH}$, and (ii) aberrant YAP activation in AH hepatocytes leads to the development of a cholangiocyte signature in hepatocytes that is involved in defective liver regeneration. These original data on the Hippo/YAP pathway in AH could provide targets for novel therapies to promote hepatocyte maturation and effective regeneration in $\mathrm{AH}$. 


\section{METHODS:}

\section{Patients}

Alcoholic hepatitis was biopsy-proven and severe forms of disease were defined by a Maddrey discriminant function $>32$. Non-response to medical therapy was defined by a Lille model score $>0.45$.

Liver samples were obtained from 23 patients who underwent early liver transplantation at Huriez Hospital's Liver Unit (Lille, France) for severe AH that did not respond to medical therapy. [5]

Since severe AH often occurs on preexisting cirrhosis, 20 patients with alcoholic cirrhosis without superimposed $\mathrm{AH}$ were used as diseased controls (Cirrh). Patients underwent liver transplantation for decompensated alcoholic cirrhosis and severe liver failure after a prolonged period of abstinence. Fragments of healthy liver samples were obtained from 20 patients who underwent liver resection for hepatic tumours (controls). The liver samples were immediately fixed or frozen for all experiments except RNA Seq. Patient characteristics are summarized in Supplementary Table 1. Study is authorized by the Lille ethical committee and informed consent was obtained from all subjects.

\section{Chronic exposure to alcohol}

Eight-week-old female mice were intravenously injected with adeno-associated viruses (AAVs): AAV2/8-YAPS127A or with an irrelevant AAV. Two weeks later, a liquid diet based on the Lieber de Carli (LDC) diet was initiated and given for 17 days [20]. The final ethanol concentration was $6.3 \% \mathrm{vol} / \mathrm{vol}$, and ethanol accounted for $28 \%$ of the total calorie content. The control diet was obtained by replacing the ethanol by an equivalent quantity of maltodextrin. Body weight and food intake were measured daily throughout the experiment. The mice were randomized into three groups: 10 mice received the control diet $(\mathrm{CD})$ and the irrelevant $\mathrm{AAV}$, 5 received the LDC diet and the irrelevant AAV, 15 received the CD and the AAV2/8- 
YAPS127A and 20 received the LDC diet and the AAV2/8-YAPS127A. The liver was removed, weighed, and either fixed in paraformaldehyde or snap-frozen in liquid nitrogen. All samples were stored at $-80^{\circ} \mathrm{C}$ until use.

\section{AAV2/8-YAPS127A preparation and purification}

The AAVs were manufactured and purified at CPV-UMR1089 (Nantes, France). Briefly, cDNA for the constitutively active human YAP was cloned from the plasmid YAPS127A (Addgene \#27370) to the pAAV2 vector plasmid (containing ITR-2 from pSub201 plasmid) using specific restriction enzymes Kpn1/EcoR5 for pAAV.CMV.YAPS127A construct and then subcloned using Not1 to obtain pAAV.LP1.YAPS127A construct. The YAPS127A transgene was under the control of the hepatocyte specific promoter LP1 [21].

Adherent HEK293 cells seeded in CellStack 5 chambers were co-transfected with the vector plasmid and helper plasmid (containing helper genes from adenovirus and the rep cap genes, depending on the capsid serotype) and harvested 3 days post-transfection. Recombinant AAV vectors were purified from supernatant PEG-precipitation by double $\mathrm{CsCl}$ gradient ultracentrifugation. The viral suspension was formulated in Dulbecco's Phosphate-Buffered Saline $1 \mathrm{X}$ buffer and stored below $-70^{\circ} \mathrm{C}$ in polypropylene low-binding cryovials. The recombinant AAV vector genome was titrated using a quantitative polymerase chain reaction. The target amplicons corresponded to inverted terminal repeats (ITR)-2, as described by D’Costa et al [22].

\section{Statistics}

Data were expressed as the mean $\pm \mathrm{SD}$ or the median. A Mann-Whitney test was used for all comparisons. Statistical analyses were performed using GraphPad Prism software (version 5.0, San Diego, CA, USA). The threshold for statistical significance was set to $\mathrm{p}<0.05$.

For further details regarding the materials and methods used, please refer to the CTAT table and supplementary materials. 


\section{RESULTS}

\section{The Hippo/YAP pathway is deregulated in the AH liver}

Liver RNA sequencing revealed that the Hippo/YAP pathway was significantly altered in AH with increased levels of $Y A P 1$ and its cofactors TEADs. The extent of alteration was associated with disease severity (Fig. 1A). PCR analysis confirmed that liver expression of YAP1 mRNA was two times higher in Cirrh and AH patients than in controls. The YAP target genes and TEAD4 were specifically induced in AH livers (Fig. 1B, Fig.S1A).

Conversely, the protein kinase MST1, a key component of the Hippo signal, was significantly lower in the AH group than in the controls and Cirrh groups and the active form of MST1 was low in AH livers, compared to Cirrh livers and control livers (Fig. 1C). These findings indicate that the Hippo signal was muted in AH livers.

Immunostaining experiments showed intense YAP staining in AH liver samples mainly located in hepatocytes throughout the parenchyma confirmed by quantification of the YAP staining in liver lobules (Fig. 1D, Fig.S1B). On the other hand, YAP expression was weak and diffuse in control livers. YAP staining was limited to the ductular reaction present in fibrotic bands surrounding the regeneration nodules in Cirrh livers while the hepatocytes were weakly stained (Fig. 1D).

\section{YAP activation in AH hepatocyte is associated with the expression of biliary markers}

Co-staining experiments showed intense YAP staining mainly in the nucleus of AH hepatocytes (albumin-positive cells) (Fig. 2A). Even if some heterogeneity in the Alb and YAP co-staining was observed in AH liver parenchyma, all patients had a majority of YAP+ cells and about one third of parenchymal cells lost their albumin expression (Fig.S2). Control and Cirrh samples only displayed weak, diffuse YAP staining in the cytoplasm of albumin-positive cells (Fig. 2A, Fig.S4). 
Biliary markers (i.e. SOX9 and HNF1 $\beta$ ) were strongly expressed in almost all hepatocytes in AH. Conversely, they were only expressed in the bile ducts of control livers and in the areas of perinodular ductular reactions (but not in hepatocytes) in Cirrh livers (Fig. 2B, Fig. S5). In AH livers, YAP and SOX9 colocalized in the hepatocyte nuclei throughout the parenchyma (Fig.S6).

In $\mathrm{AH}$ hepatocytes, abnormal expression of biliary markers was associated with low levels of hepatocyte-specific genes. Indeed, mRNA expression of the genes coding for CYP3A4, tyrosine aminotransferase (TAT), albumin and aldolase B was significantly lower in AH livers than in Cirrh and control livers (Fig. 2C).

Other Yap targets involved in the biliary commitment of liver epithelial cells [16] such as JAG1 and NOTCH2 were highly expressed in AH compared to controls and Cirrh (Fig.S7).

\section{Transcriptome analysis in microdissected tissue in AH reveals a loss of hepatocyte identity}

Specific dysregulation was identified in the transcriptome of microdissected hepatocytes (YAPpositive cells) from AH patients compared to hepatocytes (YAP-negative cells) from Cirrh and control patients (Fig. 3A). In unbiased hierarchical clustering and partial component analyses, AH hepatocytes clustered separately while Cirrh hepatocytes were distributed closer to the control samples than to AH (Fig. 3A and B). The number of differentially expressed genes was higher in AH-derived hepatocytes than in hepatocytes from controls and Cirrh (Fig. 3A). Gene set enrichment analyses (GSEAs) indicated that expression of genes related to the Hippo/YAP pathway and the downstream Notch pathway [16] was significantly enriched in microdissected cells from AH livers compared to Cirrh (Fig. 3C). AH was associated with a significant loss of hepatic identity [23] [24] gene expression and a concomitant enrichment in cholangiocyte identity genes (Fig. 3D). AH significantly exacerbated transcriptomic changes that were already present in Cirrh samples and compared to control samples (Fig.S8A). Differentially expressed genes in $\mathrm{AH}$ or Cirrh were compared with murine single-cell transcriptomic data by in silico 
mapping to study the differentiation of hepatoblasts to hepatocytes or cholangiocytes [25] (Fig.S8B). Genes that were highly expressed in AH hepatocytes corresponded to genes that are strongly expressed in hepatoblasts that differentiate into mature cholangiocytes, while genes that were highly expressed in Cirrh corresponded to genes that are highly expressed by hepatoblasts that differentiate into mature hepatocytes (Fig. 3E).

\section{Modulation of YAP drives human hepatocyte transdifferentiation}

Transfection of human primary hepatocytes with the constitutively active human YAP YAPS127A (Fig. 4A) was associated with higher mRNA expression of YAPl and its target genes, (Fig. 4B; Fig. S9A) as well as lower expression of the genes coding for hepatocyte markers CYP3A4, aldolase B, TAT and albumin (Fig. 4C), and higher levels of the biliary markers CK19 and CK7 (Fig. 4D, Fig.S9B).

Immunostaining of undifferentiated HepaRG cells just after seeding and after 15 days after confluency revealed intense nuclear YAP staining (Fig. 5A). After 30 days of culture, HepaRG cells that differentiated into biliary-type cells displayed strong YAP nuclear staining, while HepaRG cells with hepatocyte-like cell differentiation displayed weak, diffuse YAP cytoplasmic staining (Fig. 5A; Fig.S10A).

HepaRG cells treated with the YAP inhibitor, dobutamine, for 30 days led to the commitment of cells towards the hepatocytic lineage (Fig. 5B; Fig.S10B). Dobutamine treatment was associated with lower mRNA expression of YAPl and its target genes (Fig. 5C; Fig.S10C). Conversely, dobutamine treatment was associated with upregulation of the genes coding for the hepatocyte markers TAT, CYP3A4, albumin, and aldolase B (Fig. 5D), and downregulation of the biliary markers CK7 and CK19 (Fig. 5E).

Treatment of AH-isolated hepatocytes with a YAP inhibitor promotes the reversal of their transdifferentiation to cholangiocytes. 
In order to investigate the therapeutic potential of YAP inhibition on hepatocyte dedifferentiation, we have developed a protocol to isolate hepatocytes from an explant of a patient transplanted for $\mathrm{AH}$ and treated them with dobutamine for 24hrs (Fig. 6A).

Dobutamine treatment of $\mathrm{AH}$ hepatocytes decreased gene expression of YAP and more importantly of its target genes confirming at least the inhibition of its activity (Fig. 6B). This treatment significantly lowered the biliary markers expression without significant changes in hepatocyte markers expression (Fig. 6C and D).

These results suggest that YAP inhibition could limit hepatocyte defects in AH.

YAP activation induces hepatocyte transdifferentiation in animal models of alcohol exposure and impaired liver repair

Excessive alcohol intake did not appear to be sufficient to induce YAP activation in liver. We then evaluated potential in vivo interactions between uncontrolled YAP activation in hepatocyte and alcohol intake using a model of Lieber de Carli.

To this aim, YAP activation was investigated in the hepatocytes of mice exposed to alcohol, after injection of an adeno-associated virus (AAV) with a hepatic tropism overexpressing mutated human YAP (AAV-YAPS127A).

Mice fed with either ethanol alone (LDC) or exposed to ethanol and AAV-YAPS127A (YAPS127A-LDC) displayed a significant increase in the liver steatosis and in the liver weight to body weight ratio as compared to mice fed with control diet (CD) (Fig.S11A-C). Transduction efficacy was confirmed by the expression of human mutated YAP in the mouse livers after AAV-YAPS127 treatment (Fig.S11D).

Immunostaining of YAP revealed that in vivo transduction of YAPS127A leads to induction of YAP expression and its nuclear localization in hepatocytes (Fig. 7A). Livers of CD and LDC 
mice showed YAP staining in intrahepatic bile cells with weak and diffuse staining in the hepatic parenchyma (Fig. 7A).

An abnormal SOX9 staining was observed in hepatocytes of YAPS127A-LDC mice as compared to $\mathrm{CD}$ and LDC mice where SOX9 staining was restricted to intrahepatic bile cells and compared YAPS127A-CD where limited DR is stained (Fig. 7A). This was also associated with a significant induction of liver mRNA expression of $\operatorname{Sox} 9$ and $H n f 1 \beta$, two biliary genes, in YAPS127A-LDC mice as compared to other group of mice (Fig. 7B).

Unexpectedly, liver of YAPS127A-LDC mice displayed large areas of necrosis and significant neutrophil infiltration in the hepatic parenchyma, which was not observed in CD, YAPS127ACD and LDC mice (Fig. 7C). This cellular inflammation was associated with a significant induction of mRNA expression of the inflammatory cytokines IL1 $\beta$ and TNF $\alpha$ in the liver of YAPS127A-LDC mice as compared to the CD, YASPS127A-CD and LDC livers (Fig. 7D). These results suggested that YAP activation in hepatocyte together with alcohol exposure induced a rapid hepatocyte transdifferentiation associated with liver inflammation and necrosis, common features characterizing AH.

Hepatic regeneration defect is also a key feature in $\mathrm{AH}$ pathophysiology, we have evaluated the consequences of the constitutive uncontrolled hepatocyte activation of YAP (using AAVYAPS127A) on hepatic repair in a validated mouse model of $\mathrm{CCl}_{4}$ injury. We first verified that YAP activation did not influence $\mathrm{CCl}_{4}$ metabolism and induced necrosis (Fig.S12).

Forty-eight hours after $\mathrm{CCl}_{4}$ injection, the livers of mice transduced with YAPS127A $\left(\mathrm{CCl}_{4}\right.$ +YAPS127A) exhibited a 77\% decrease in the number of BrdU-positive (proliferating) hepatocytes compared to control mice $\left(\mathrm{CCl}_{4}\right)$ (Fig. 7E and F). This lack of proliferative response is not due to differences in $\mathrm{CCl}_{4}$-induced injury and metabolism in both groups (Fig.S12). As observed in the LDC model, this regeneration defect was associated with the induction of the 
liver mRNA expression of biliary markers, $\mathrm{Hnfl} \beta$ and Sox9 in $\mathrm{CCl}_{4}+\mathrm{YAPS} 127 \mathrm{~A}$ mice as compared to $\mathrm{CCl}_{4}$ mice (Fig. 7G). These results suggest that the uncontrolled activation of a YAP in hepatocyte after injury profoundly affects the regenerative capacity of hepatocytes in favour of their transdifferentiation toward a biliary phenotype. 


\section{DISCUSSION}

The development of novel pathophysiological-based drugs is needed to improve the outcome of the life-threatening disease, alcoholic hepatitis [1]. The present study provides convincing evidence that in severe $\mathrm{AH}$, activation of the Hippo/YAP pathway in hepatocytes leads to a progressive shift from a hepatocyte identity to a biliary fate. This shift in differentiation is confirmed in vivo and is found to be associated with the blockade of hepatocyte regeneration. Finally, ex vivo experiments using $\mathrm{AH}$ hepatocytes provide additional evidence that a YAP inhibitor could be a targeted therapeutic approach in severe $\mathrm{AH}$.

Most data on the potential pathophysiological role of Hippo/YAP have been obtained in animal models, while functional data in humans are scarce. Our comprehensive study demonstrates that unregulated YAP activation is a key mechanism in the transdifferentiation of hepatocytes into biliary cells in the adult human liver. This is in line with recent data obtained in acute liver failure, where injury-related activation of YAP was observed in accumulating foetal-like hepatocytes [26]. In AH, YAP expression is inversely correlated to the expression of the epithelial splicing regulatory protein-2 (ESRP2) [27]. ESRP2 suppression has been shown to be associated with adult to foetal reprogramming and proliferative response in mice. The foetal isoform of an essential liver-enriched transcription factor (HNF4 $\alpha)$ has also been shown to be strongly expressed in AH livers [28]. It is interesting to note that in mice, YAP has been shown to deregulate transcriptional programs known to control hepatocyte quiescence and differentiation induced by HNF4A [29]. Our study in human samples identifies an additional phenomenon: the transdifferentiation of hepatocytes resulting in the loss of their cellular identity and function.

The low MST1 kinase activity is a strong evidence of Hippo silencing in AH leading to YAP activation. To better understand the aberrant YAP activation in hepatocytes, we evaluated 
GSEAs in microdissected AH hepatocytes, and found that they displayed an enriched biliary gene signature and marked downregulation of hepatocyte identity genes.

Hepatocyte microdissection clearly demonstrates the presence of a severe defect in proliferation and metabolic function in hepatocytes in $\mathrm{AH}$. On the other hand, in cirrhosis without $\mathrm{AH}$, hepatocytes retain most of their physiological function compared to control hepatocytes. These data show the specific fate of hepatocytes in $\mathrm{AH}$ and strongly support the need for targeted therapies.

Several reports described in the rodent model of hepatectomy, the importance of a transient activation peak of YAP in early phases of liver regeneration [14]. YAP has then been shown to promote early hepatocyte cell cycle progression [30]. This is in contrast with the present data showing that YAP activation blocked hepatocyte repair. It is important to consider that the influence of transient activation of YAP could differ from that of sustained activation. Indeed, sustained unregulated activation of YAP in murine hepatocytes leads to their transdifferentiation into epithelial cells with an intermediate phenotype [16] [18] rather than to their proliferation. The fact that YAP activation is sustained and unregulated in AH could play a role in defective hepatic repair because hepatocytes are already engaged in a process of transdifferentiation as shown in our $\mathrm{CCl}_{4}$ experiments. Thus, further studies are needed to clarify the role of YAP and its partners in liver regeneration in the context of hepatocyte transdifferentiation.

According to the literature, alcohol consumption is not sufficient to recapitulate AH pattern (inflammatory infiltrates, necrosis, hepatocyte transdifferentiation...) in animal models. In the present study, we have coupled YAP activation in hepatocyte and alcohol intake and demonstrate a rapid trandifferentiation of hepatocytes associated liver necrosis and obvious inflammatory infiltrates. This combination of features characteristic for $\mathrm{AH}$ were virtually never described in animal models of ALD. 
The Hippo/YAP pathway plays a complex role in cell proliferation, organ regeneration, and carcinogenesis. Fifteen years ago, the YAP genomic locus was found to be amplified in breast and liver cancers [31] [32] and experiments in models of chemical injury-induced hepatocellular carcinoma have suggested that elevated YAP activity may be an early oncogenic event. Indeed, nuclear YAP was found in preneoplastic hepatic foci, and the number and size of tumours was markedly lower when YAP was targeted [33]. In $\mathrm{AH}$, the competing risk of liver-failure-related mortality probably excludes mid- or long-term oncogenic effects of YAP activation. Indeed, patients with AH either die within 6 months due to liver failure or they show a marked improvement in liver function, which probably results in the reversion of YAP activation in hepatocytes.

This study provides an interesting opportunity for drug development in AH. Pharmaceutical companies are increasingly interested in developing drugs targeting the Hippo/YAP pathway because of the potential benefits on cell proliferation, cell transdifferentiation, and tissue regeneration [34]. Further preclinical studies must evaluate whether YAP-interfering agents have beneficial effects in ALD.

In conclusion, the present study demonstrates that sustained aberrant activation of the Hippo/YAP pathway in hepatocytes plays a role in hepatocyte transdifferentiation in $\mathrm{AH}$, leading to a loss of hepatocyte identity and impaired liver regeneration. Considering the limited number of therapeutic options in $\mathrm{AH}$, targeting YAP is a promising strategy for the treatment of patients with this disease. 
List of abbreviations: alcoholic hepatitis (AH), alcohol-associated liver disease (ALD), adenoassociated virus (AAV), carbon tetrachloride $\left(\mathrm{CCl}_{4}\right)$, control diet $(\mathrm{CD})$, false discovery rate (FDR), gene set enrichment analysis (GSEA), hepatic progenitor cell (HPC), Lieber-De Carli (LDC), phosphate-buffered saline (PBS), principal component analysis (PCA), paraformaldehyde (PFA), polymorphonuclear neutrophil (PMN), reverse transcriptase polymerase chain reaction (RT-PCR), wild type (WT), Yes-associated protein (YAP).

Acknowledgements: The authors thank the Centre de Production de Vecteurs facility (INSERM U1089, Nantes, France) for production of the AAVs. We also thank Biopredic International for their collaboration and training in the isolation and culture of primary human hepatocytes and the immunohistochemistry laboratory at the Center for Biology - Pathology (Lille University Medical Center), more particularly Nicolas Van Pouck for excellent technical support. We are grateful to Jonathan Vanhoutte for set-up assistance and expert technical support for laser-capture microdissection. Sequencing was performed by the GenomEast platform, a member of the "France Génomique" consortium (ANR-10-INBS-0009). From GenomEast we would like to particularly thank Christelle Thibault-Carpentier for the RNASeq experiments, and Matthieu Jung for expert biostatistics analysis. The authors would like to thank Antonino Bongiovanni and Meryem Tardivel from the BioImaging Center Lille (F-59000 Lille, France) for the access and excellent technical support. 


\section{REFERENCES:}

[1] Mandrekar P, Bataller R, Tsukamoto H, Gao B. Alcoholic hepatitis: Translational approaches to develop targeted therapies. Hepatology 2016;64:1343-55. https://doi.org/10.1002/hep.28530.

[2] Lucey MR, Mathurin P, Morgan TR. Alcoholic hepatitis. N Engl J Med 2009;360:275869. https://doi.org/10.1056/NEJMra0805786.

[3] Louvet A, Thursz MR, Kim DJ, Labreuche J, Atkinson SR, Sidhu SS, et al. Corticosteroids Reduce Risk of Death Within 28 Days for Patients With Severe Alcoholic Hepatitis, Compared With Pentoxifylline or Placebo-a Meta-analysis of Individual Data From Controlled Trials. $\quad$ Gastroenterology 2018;155:458-468.e8. https://doi.org/10.1053/j.gastro.2018.05.011.

[4] Louvet A, Naveau S, Abdelnour M, Ramond M-J, Diaz E, Fartoux L, et al. The Lille model: A new tool for therapeutic strategy in patients with severe alcoholic hepatitis treated with steroids. Hepatology 2007;45:1348-54. https://doi.org/10.1002/hep.21607.

[5] Mathurin P, Samuel D, Durand F, Pageaux G-P, Dharancy S, Boleslawski E, et al. Early Liver Transplantation for Severe Alcoholic Hepatitis. N Engl J Med 2011:11.

[6] Adachi Y, Moore LE, Bradford BU, Gao W, Thurman RG. Antibiotics prevent liver injury in rats following long-term exposure to ethanol. Gastroenterology 1995;108:218-24. https://doi.org/10.1016/0016-5085(95)90027-6.

[7] Iimuro Y, Gallucci RM, Luster MI, Kono H, Thurman RG. Antibodies to tumor necrosis factor alfa attenuate hepatic necrosis and inflammation caused by chronic exposure to ethanol in the rat. Hepatol Baltim Md 1997;26:1530-7. https://doi.org/10.1002/hep.510260621. 
[8] Yin M, Wheeler MD, Kono H, Bradford BU, Gallucci RM, Luster MI, et al. Essential role of tumor necrosis factor alpha in alcohol-induced liver injury in mice. Gastroenterology 1999;117:942-52. https://doi.org/10.1016/s0016-5085(99)70354-9.

[9] Louvet A, Mathurin P. Alcoholic liver disease: mechanisms of injury and targeted treatment. Nat Rev Gastroenterol Hepatol 2015;12:231-42. https://doi.org/10.1038/nrgastro.2015.35.

[10] Sancho-Bru P, Altamirano J, Rodrigo-Torres D, Coll M, Millán C, José Lozano J, et al. Liver progenitor cell markers correlate with liver damage and predict short-term mortality in patients with alcoholic hepatitis. Hepatology 2012;55:1931-41. https://doi.org/10.1002/hep.25614.

[11] Odena G, Chen J, Lozano JJ, Altamirano J, Rodrigo-Torres D, Affo S, et al. LPS-TLR4 Pathway Mediates Ductular Cell Expansion in Alcoholic Hepatitis. Sci Rep 2016;6:35610. https://doi.org/10.1038/srep35610.

[12] Dubuquoy L, Louvet A, Lassailly G, Truant S, Boleslawski E, Artru F, et al. Progenitor cell expansion and impaired hepatocyte regeneration in explanted livers from alcoholic hepatitis. Gut 2015;64:1949-60. https://doi.org/10.1136/gutjnl-2014-308410.

[13] Fan F, He Z, Kong L-L, Chen Q, Yuan Q, Zhang S, et al. Pharmacological targeting of kinases MST1 and MST2 augments tissue repair and regeneration. Sci Transl Med 2016;8:352ra108-352ra108. https://doi.org/10.1126/scitranslmed.aaf2304.

[14] Moya IM, Halder G. Hippo-YAP/TAZ signalling in organ regeneration and regenerative medicine. Nat Rev Mol Cell Biol 2019;20:211-26. https://doi.org/10.1038/s41580-0180086-y.

[15] Zhang N, Bai H, David KK, Dong J, Zheng Y, Cai J, et al. The Merlin/NF2 tumor suppressor functions through the YAP oncoprotein to regulate tissue homeostasis in mammals. Dev Cell 2010;19:27-38. https://doi.org/10.1016/j.devcel.2010.06.015. 
[16] Yimlamai D, Christodoulou C, Galli GG, Yanger K, Pepe-Mooney B, Gurung B, et al. Hippo pathway activity influences liver cell fate. Cell 2014;157:1324-38. https://doi.org/10.1016/j.cell.2014.03.060.

[17] Planas-Paz L, Sun T, Pikiolek M, Cochran NR, Bergling S, Orsini V, et al. YAP, but Not RSPO-LGR4/5, Signaling in Biliary Epithelial Cells Promotes a Ductular Reaction in Response to Liver Injury. Cell Stem Cell 2019;25:39-53.e10. https://doi.org/10.1016/j.stem.2019.04.005.

[18] Patel SH, Camargo FD, Yimlamai D. Hippo Signaling in the Liver Regulates Organ Size, Cell Fate, and Carcinogenesis. Gastroenterology 2017;152:533-45. https://doi.org/10.1053/j.gastro.2016.10.047.

[19] Zeng Q, Hong W. The emerging role of the hippo pathway in cell contact inhibition, organ size control, and cancer development in mammals. Cancer Cell 2008;13:188-92. https://doi.org/10.1016/j.ccr.2008.02.011.

[20] Louvet A, Teixeira-Clerc F, Chobert M-N, Deveaux V, Pavoine C, Zimmer A, et al. Cannabinoid CB2 receptors protect against alcoholic liver disease by regulating Kupffer cell polarization in mice. Hepatology 2011;54:1217-26. https://doi.org/10.1002/hep.24524.

[21] Osman E, Evans V, Graham IR, Athanasopoulos T, McIntosh J, Nathwani AC, et al. Preliminary evaluation of a self-complementary AAV2/8 vector for hepatic gene transfer of human apoE3 to inhibit atherosclerotic lesion development in apoE-deficient mice. Atherosclerosis 2009;204:121-6. https://doi.org/10.1016/j.atherosclerosis.2008.08.043.

[22] D’Costa S, Blouin V, Broucque F, Penaud-Budloo M, François A, Perez IC, et al. Practical utilization of recombinant AAV vector reference standards: focus on vector genomes titration by free ITR qPCR. Mol Ther Methods Clin Dev 2016;5:16019. https://doi.org/10.1038/mtm.2016.19. 
[23] Dubois V, Gheeraert C, Vankrunkelsven W, Dubois-Chevalier J, Dehondt H, BobowskiGerard M, et al. Endoplasmic reticulum stress actively suppresses hepatic molecular identity in damaged liver. Mol Syst Biol 2020;16. https://doi.org/10.15252/msb.20199156.

[24] Verhulst S, Roskams T, Sancho-Bru P, van Grunsven LA. Meta-Analysis of Human and Mouse Biliary Epithelial Cell Gene Profiles. Cells 2019;8. https://doi.org/10.3390/cells8101117.

[25] Yang L, Wang W-H, Qiu W-L, Guo Z, Bi E, Xu C-R. A single-cell transcriptomic analysis reveals precise pathways and regulatory mechanisms underlying hepatoblast differentiation. $\quad$ Hepatol $\quad$ Baltim 2017;66:1387-401. https://doi.org/10.1002/hep.29353.

[26] Hyun J, Oh S-H, Premont RT, Guy CD, Berg CL, Diehl AM. Dysregulated activation of fetal liver programme in acute liver failure. Gut 2019;68:1076-87. https://doi.org/10.1136/gutjnl-2018-317603.

[27] Hyun J, Sun Z, Ahmadi AR, Bangru S, Chembazhi UV, Du K, et al. Epithelial splicing regulatory protein 2-mediated alternative splicing reprograms hepatocytes in severe alcoholic hepatitis. J Clin Invest 2020. https://doi.org/10.1172/JCI132691.

[28] Argemi J, Latasa MU, Atkinson SR, Blokhin IO, Massey V, Gue JP, et al. Defective HNF4alpha-dependent gene expression as a driver of hepatocellular failure in alcoholic hepatitis. Nat Commun 2019;10. https://doi.org/10.1038/s41467-019-11004-3.

[29] Fitamant J, Kottakis F, Benhamouche S, Tian HS, Chuvin N, Parachoniak CA, et al. YAP Inhibition Restores Hepatocyte Differentiation in Advanced HCC, Leading to Tumor Regression. Cell Rep 2015;10:1692-707. https://doi.org/10.1016/j.celrep.2015.02.027.

[30] Tschuor C, Kachaylo E, Ungethüm U, Song Z, Lehmann K, Sánchez-Velázquez P, et al. Yes-associated protein promotes early hepatocyte cell cycle progression in regenerating 
liver after tissue loss. FASEB BioAdvances 2019;1:51-61. https://doi.org/10.1096/fba.1023.

[31] Overholtzer M, Zhang J, Smolen GA, Muir B, Li W, Sgroi DC, et al. Transforming properties of YAP, a candidate oncogene on the chromosome 11q22 amplicon. Proc Natl Acad Sci U S A 2006;103:12405-10. https://doi.org/10.1073/pnas.0605579103.

[32] Zender L, Spector MS, Xue W, Flemming P, Cordon-Cardo C, Silke J, et al. Identification and validation of oncogenes in liver cancer using an integrative oncogenomic approach. Cell 2006;125:1253-67. https://doi.org/10.1016/j.cell.2006.05.030.

[33] Perra A, Kowalik MA, Ghiso E, Ledda-Columbano GM, Di Tommaso L, Angioni MM, et al. YAP activation is an early event and a potential therapeutic target in liver cancer development. J Hepatol 2014;61:1088-96. https://doi.org/10.1016/j.jhep.2014.06.033.

[34] Yimlamai D, Fowl BH, Camargo FD. Emerging evidence on the role of the Hippo/YAP pathway in liver physiology and cancer. J Hepatol 2015;63:1491-501. https://doi.org/10.1016/j.jhep.2015.07.008. 


\section{FIGURE LEGENDS}

Fig. 1: Abnormal YAP activation in liver samples from patients with AH.

(A) A heat map showing mRNA expression levels of Hippo/YAP pathway components and their partners in $\mathrm{AH}$ liver explants (exAH, in purple), liver biopsies from patients with severe $\mathrm{AH}$ (red), liver biopsies from patients with early-stage $\mathrm{AH}(\mathrm{eASH}$, in blue) and liver biopsies from patients with normal livers ( $\mathrm{N}$, in green). The fold change is the relative variation of gene expression to the centre value (mean of all values of that gene in the population) (B) Real-time PCRs performed on liver samples from control patients (Ctrl), patients with alcoholic hepatitis (AH) and patients with alcoholic cirrhosis (Cirrh). mRNA expression levels of $Y A P 1$, its target genes (NUAK2, ANKRD1, and TGFB2) and its cofactor TEAD4 were compared with the TBP housekeeping gene (coding for TATA-binding protein). (C) A representative Western blot of active and inactive MST1 in the liver. Beta-actin was used as a loading control. The dot plots illustrate the band intensity for total MST1 and active MST1. The results are expressed as total MST1/ $\beta$-actin and active MST1/ $\beta$-actin ratios, in relative units. (D) Representative YAP immunostaining experiments in livers from control ( $\mathrm{Ctrl})$, alcoholic hepatitis $(\mathrm{AH})$ and cirrhosis (Cirrh) groups at a magnification of x100 (upper panel) or x200 (lower panel). In control livers, the portal bile ducts were stained (arrowheads, left panel). In cirrhotic liver samples (Cirrh), we observed intense staining in the fibrotic bands around regenerating nodules. The AH liver displayed very intense staining throughout the entire parenchyma. Statistical significances are indicated (Mann-Whithney U-test).

Fig. 2: YAP is expressed in the nucleus of hepatocytes of patients with $A H$ and is associated with the expression of biliary markers by hepatocytes.

(A) Co-immunostaining of YAP (in green) and the hepatocyte marker, albumin (in red), in the livers of control patients (Ctrl), patients with $\mathrm{AH}$ and patients with alcoholic cirrhosis (Cirrh) (magnification $\times 100$ ). (B) Representative immunostaining experiments for SOX9 and HNF1 $\beta$ 
in livers from control (Ctrl), AH and cirrhosis (Cirrh) samples $(\times 200)$. In control livers the portal bile ducts were stained. In cirrhotic liver samples, intense staining is observed in the fibrotic string around regenerating nodules. The $\mathrm{AH}$ liver displayed intense staining in hepatocytes throughout the parenchyma (arrow heads). (C) Real-time PCR, shows that the low levels of mRNA expression of hepatocyte markers (genes coding for CYP3A4, TAT, albumin and aldolase B) are specific for AH liver samples, compared to cirrhosis (Cirrh) and control (Ctrl) liver samples. Statistical significances are indicated (Mann-Whithney U-test).

Fig. 3: Hepatocyte-to-cholangiocyte changes in cell identity, as revealed by transcriptomic analyses.

(A) Coloured Heat map of sample-to-sample distances displaying hierarchical clustering. The Simple Error Ratio Estimate (SERE) coefficient that quantifies global RNA-seq sample differences has been used. The higher SERE is, the more the samples are different. The number of differentially expressed genes ( $\log _{2}$ fold change $>1$; FDR $<0.05$ ) in pairwise comparisons was used to generate a grayscale heat map. (B) A PCA of normalized gene expression data from microdissected hepatocytes from AH, Cirrh and Ctrl patients. (C) Enrichment plots from GSEAs of genes related to the Hippo/YAP or Notch pathways as indicated, and transcriptomic differences between $\mathrm{AH}$ and Cirrh samples. (D) GSEAs similar to those in (C) were used to define a hepatocyte identity (Hep-ID) or a cholangiocyte identity (Chol-ID), as indicated. (E) Mean expression levels of genes identified as being significantly more expressed in single cells from the hepatobiliary lineage in Cirrh or AH samples. Details of the data processing steps are given in the Methods section and Fig S3. The hepatoblast-to-hepatocyte and hepatoblast-tocholangiocyte differentiation pathways are indicated.

Fig. 4: Yap modulation in human primary hepatocytes affects the hepatocyte phenotype.

(A) A schematic illustration of the nucleofection protocol for the YAPS127A expression vector in human primary hepatocytes. (B) After 7 days of culture, the mRNA expression levels of 
$Y A P 1$, its target genes (ANKRD1, NUAK2, TGF 2 2, NOTCH2 and $J A G 1)$, (C) genes coding for hepatocyte markers (CYP3A4, aldolase B, TAT and albumin) and (D) biliary markers ( $C K 7$ and CK19) were evaluated in mock-transfected hepatocytes (white bars) or YAPS127A-transfected hepatocytes (black bars). The results are expressed in relative units (mean $\pm \mathrm{SEM})$. Statistical significances are indicated (Mann-Whithney U-test).

Fig. 5: Inhibition of YAP in HepaRG cells favours their differentiation into hepatocytes.

(A) Green immunofluorescent staining of YAP in undifferentiated HepaRG cells (HepaRG D0, HepaRG D15) and differentiated HepaRG cells (HepaRG D30). Red arrowheads indicate the absence of nuclear YAP staining in hepatocyte-like cells $(\mathrm{H})$. Biliary-type cells $(\mathrm{CH})$ displayed substantial YAP staining in the nucleus. (B) A representative picture of the HepaRG cells phenotype after a 30-day differentiation period in cells treated with the vehicle (HepaRG+vehicle) or the YAP inhibitor dobutamine (HepaRG+dobutamine). Dashed lines delineate areas of HepaRG differentiated into hepatocytes (H). (C) A histogram showing mRNA expression levels of $Y A P 1$, its target genes (ANKRD1, NUAK2, TGFß2, NOTCH2 and $J A G 1$ ). (D) A histogram showing mRNA expression levels of genes coding for hepatocyte markers (CYP3A4, aldolase B, TAT and albumin). (E) biliary markers ( $C K 7$ and $C K 19)$. The results are expressed in relative units (mean $\pm \mathrm{SD})$, and the statistical significances are indicated (Mann-Whithney U-test).

Fig. 6: Dobutamine treatment of primary AH hepatocytes reverts their transdifferentiation into biliary cells

(A) A schematic illustration of the protocol for the isolation and culture of primary hepatocytes from $\mathrm{AH}$ livers and treated with dobutamine or vehicle (PBS) for 24hrs. (B) A histogram representing mRNA expression of $Y A P 1$, its target genes (ANKRD1, NUAK2, and TGFB2). (C) A histogram representing mRNA expression of genes coding for hepatocyte markers (CYP3A4, aldolase $\mathrm{B}, T A T$, and albumin). (D) A histogram representing mRNA expression of biliary 
markers ( $H N F 1 \beta, S O X 9, C K 7$ and $C K 19)$. The results are expressed in relative units (mean $\pm \mathrm{SD})$ and statistical significances are indicated (Mann-Whithney U-test).

Fig. 7: In vivo activation of YAP in hepatocytes led to transdifferentiation upon alcohol exposure, and blocked hepatocyte proliferation after $\mathrm{CCl}_{4}$-induced injury.

(A) Representative YAP and SOX9 immunostaining experiments (magnification x200). (B) A histogram representing liver Sox9 and Hnf1 $\beta$ mRNA expression in livers. The results are expressed in relative units (mean $\pm \mathrm{SEM}$ ), and the statistical significance is indicated. (C) Representative H\&E histological picture of livers obtained from CD, LDC, YAPS127A-CD and YAPS127A-LDC mice (x200). Yellow arrowheads indicate the inflammatory infiltrates and dashed lines delineate areas of liver necrosis. (D) A histogram representing liver Tnf $\alpha$ and Il- $1 \beta$ mRNA expression in livers. The results are expressed in relative units (mean $\pm \mathrm{SEM}$ ), and the statistical significances are indicated (Mann-Whithney U-test).

(E) Immunostaining evaluating BrdU incorporation $48 \mathrm{~h}$ after $\mathrm{CCl}_{4}$ intoxication in mice treated with $\mathrm{CCl}_{4}$ and injected with an irrelevant AAV $\left(\mathrm{CCl}_{4}\right)$ or AAV2/8-YAPS127A $\left(\mathrm{CCl}_{4}+\right.$ YAPS127A). Arrowheads: BrdU-positive hepatocytes. (F) A histogram representing the BrdU-positive cell count $48 \mathrm{~h}$ after $\mathrm{CCl}_{4}$ injection in mice. The results are expressed as the mean \pm SD number of BrdU-positive hepatocytes per $\mathrm{mm}^{2}$. (G) A histogram representing liver Sox9 and Hnfl $\beta$ mRNA expression in mice livers. The results are expressed in relative units (mean $\pm \mathrm{SD}$ ), and the statistical significances are indicated (Mann-Whithney U-test). 
Figure 2

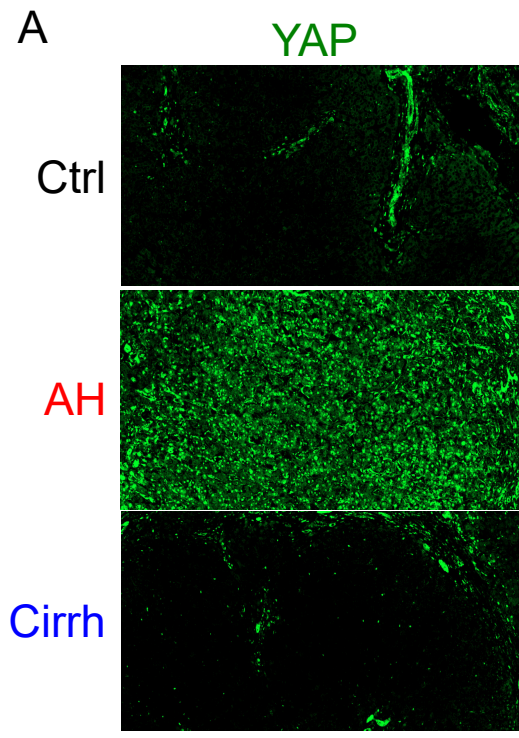

Albumin

Merge

B
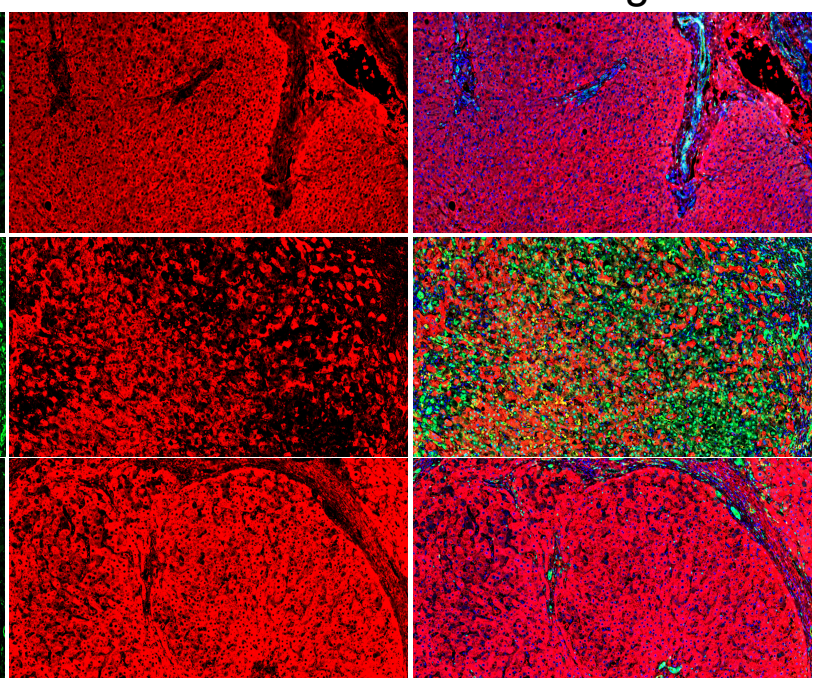

SOX9

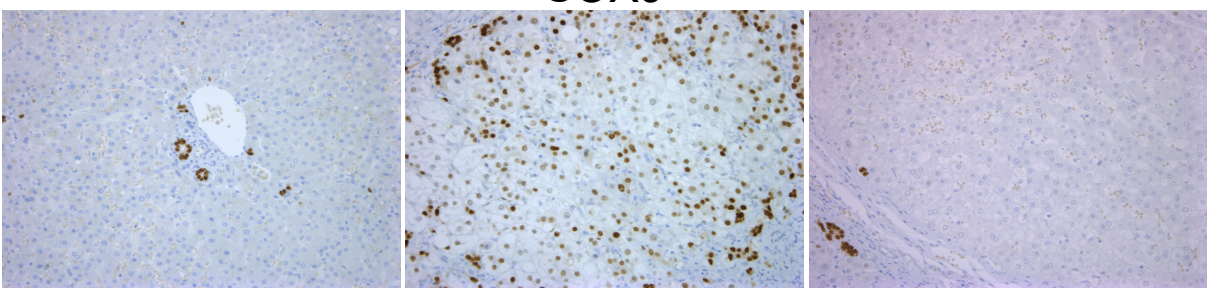

HNF1 $\beta$

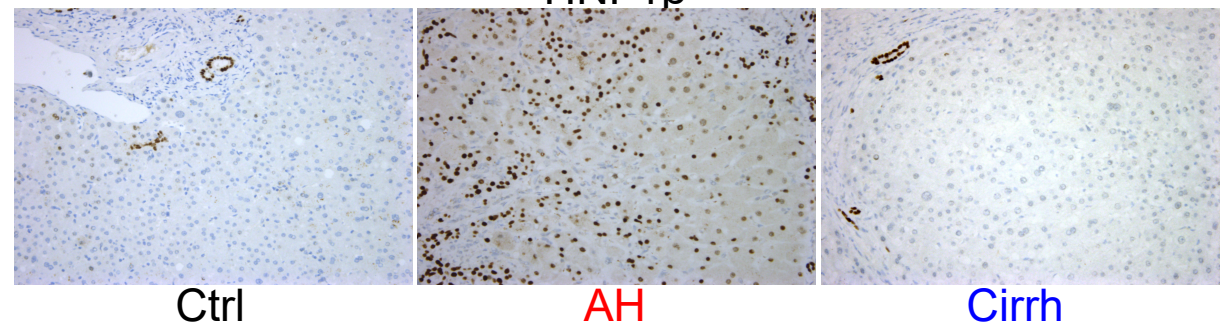

C
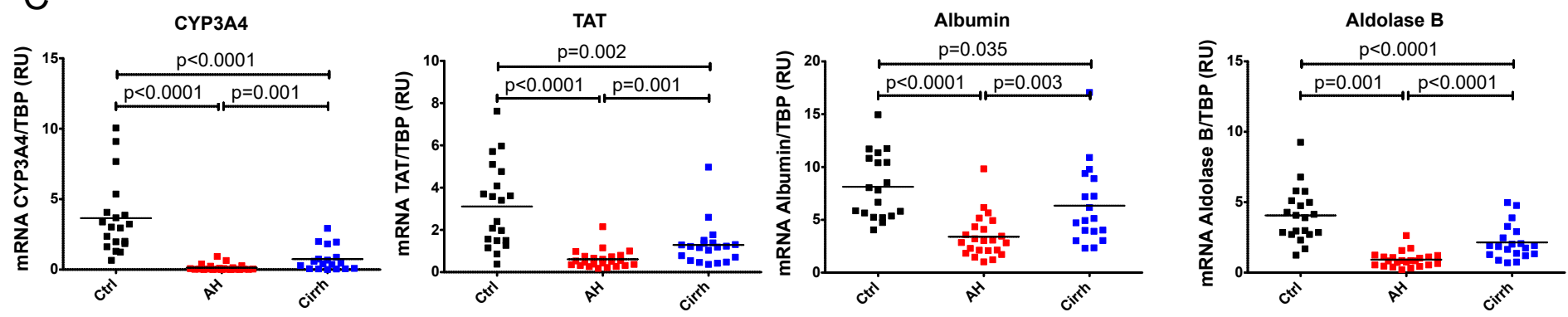
Figure 3
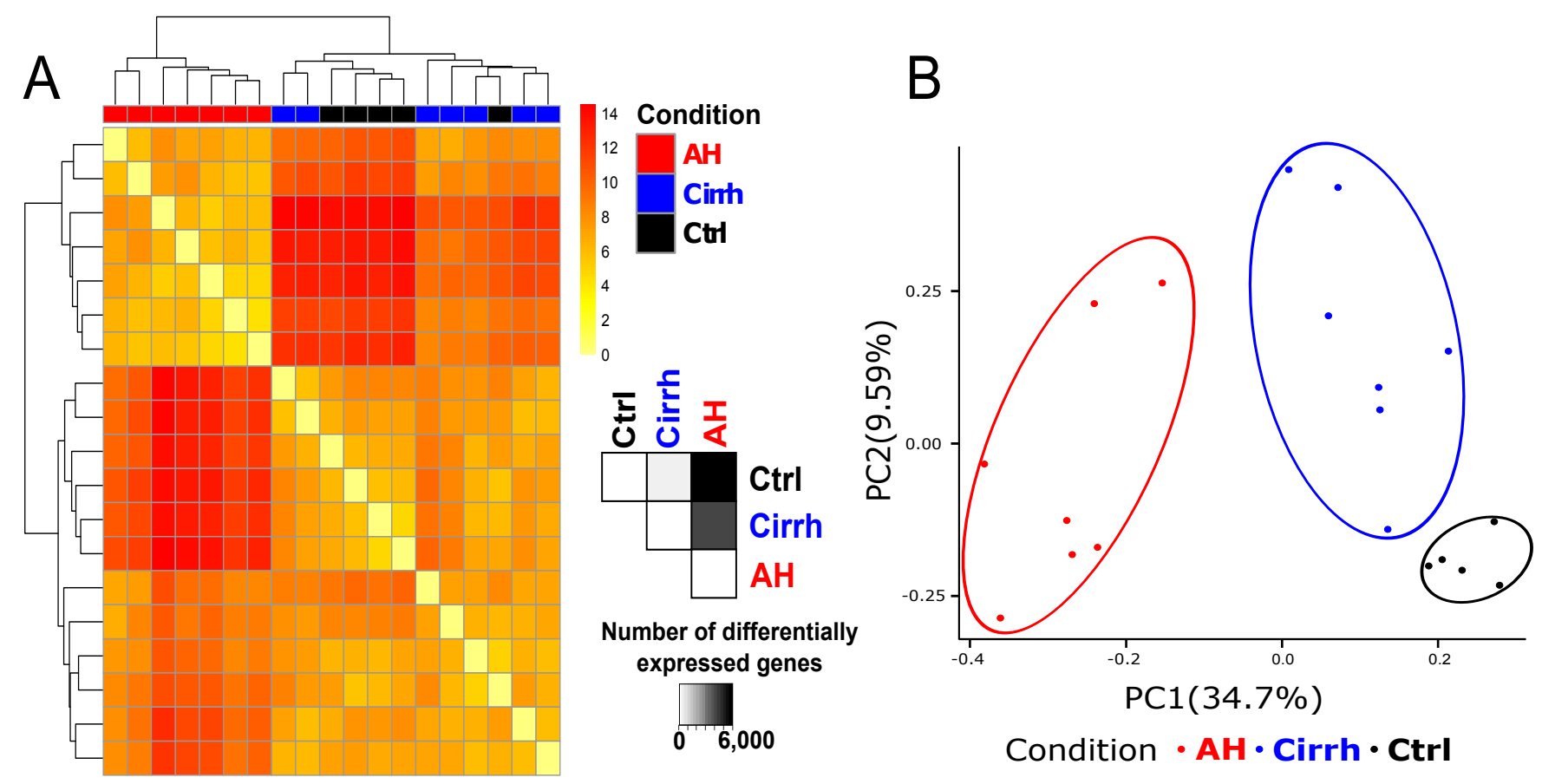

C
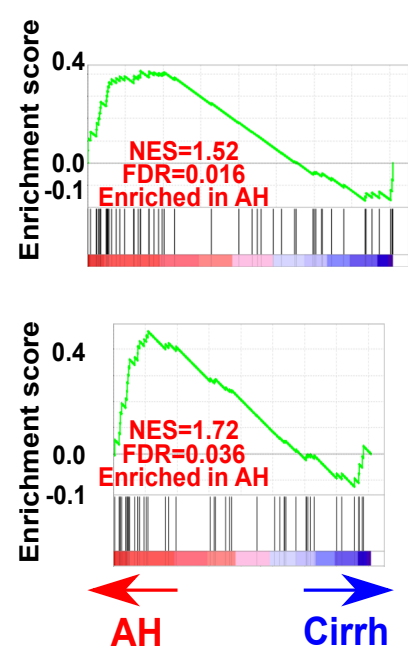

HIPPO/YAP signaling

NOTCH signaling
D
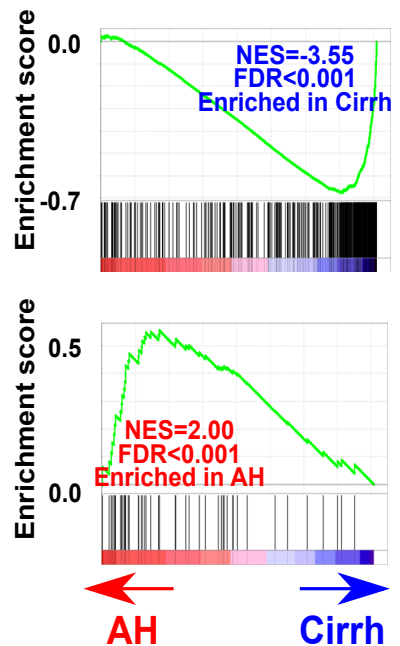

E

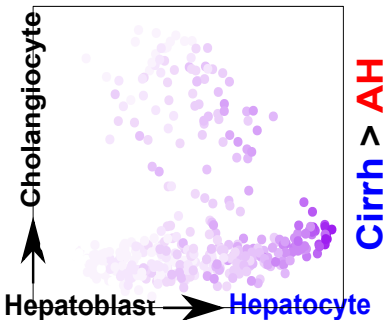

Chol-ID

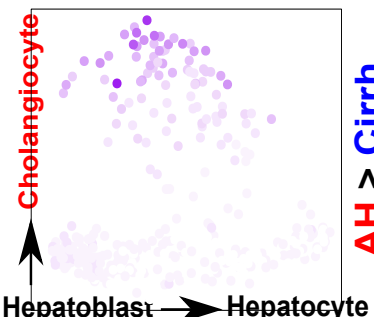


Figure 4
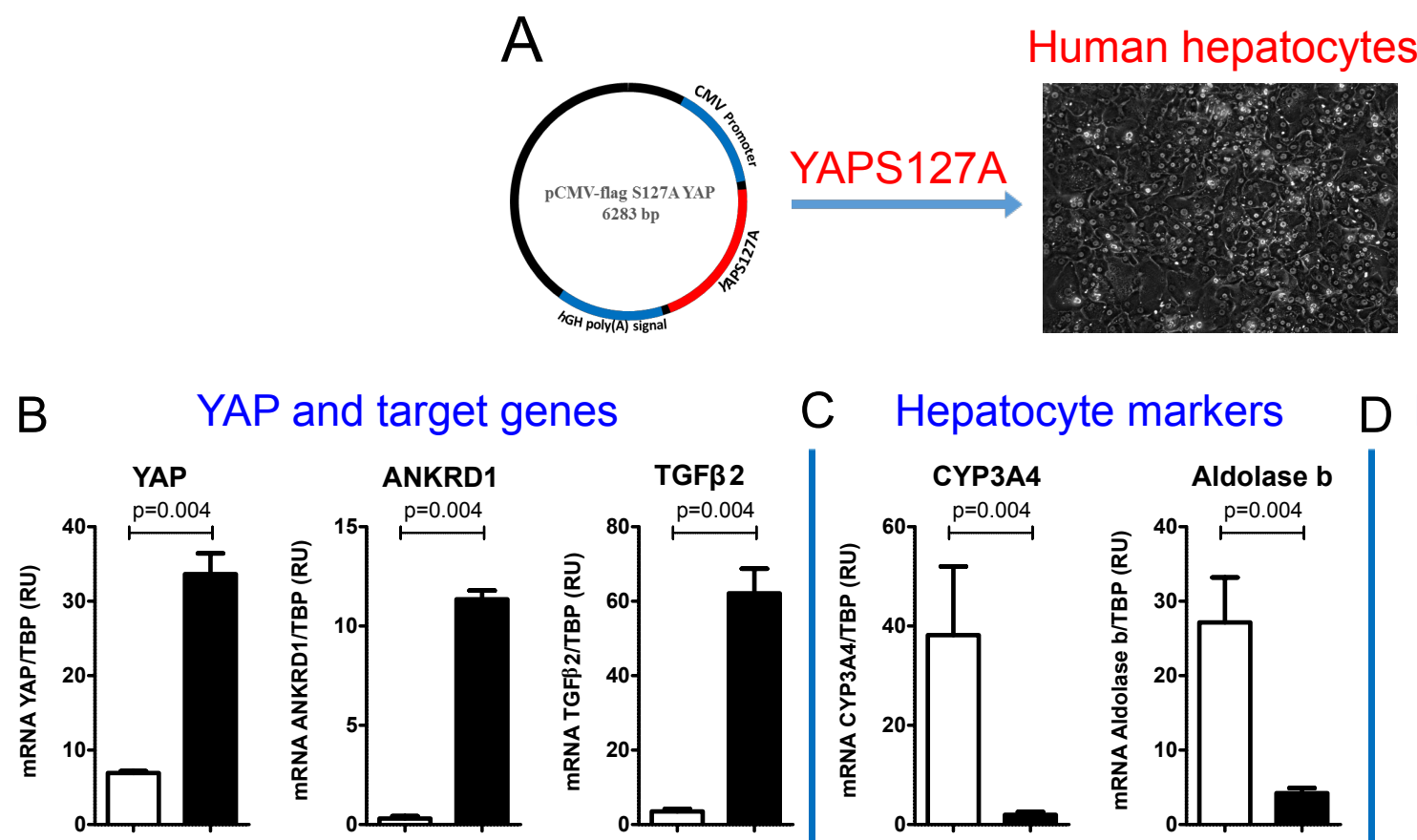

C Hepatocyte markers

D Billiary markers
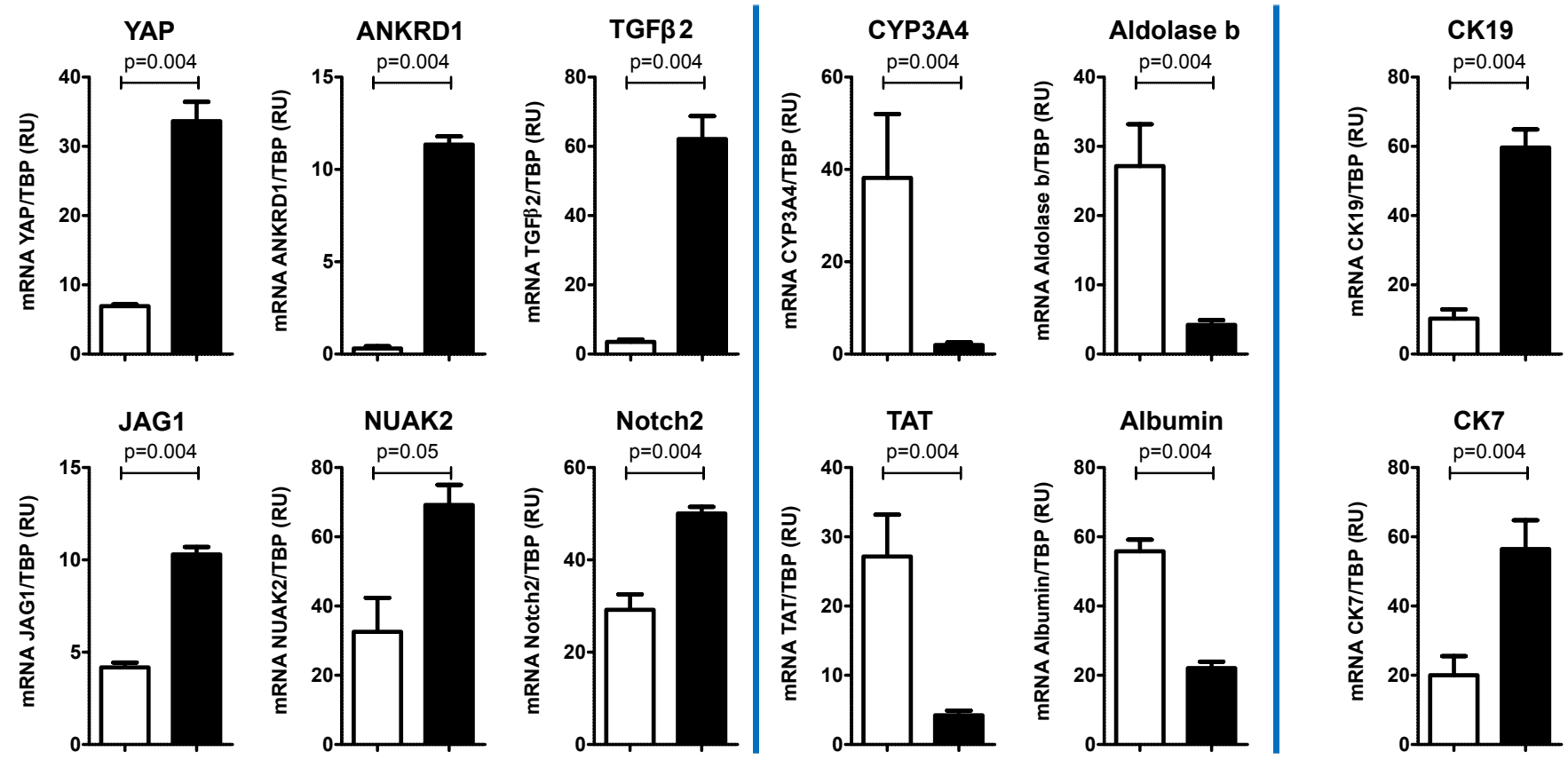

Hepatocyte mock-transfected

Hepatocyte transfected with YAPS127A 
Figure 5

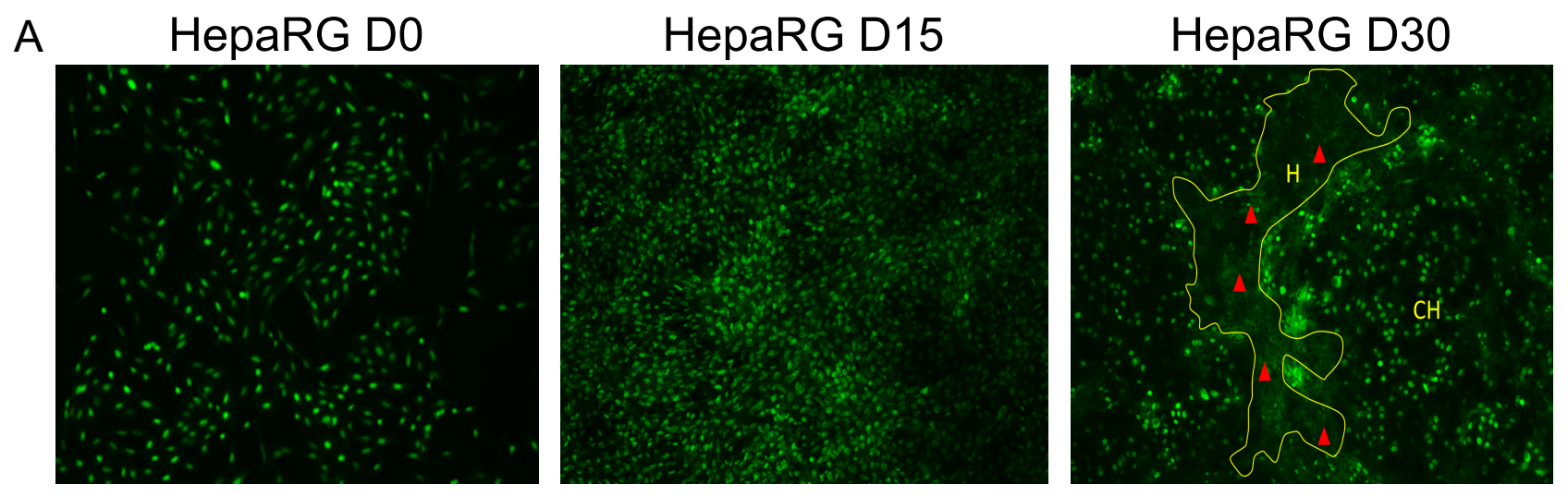

B

HepaRG + vehicle

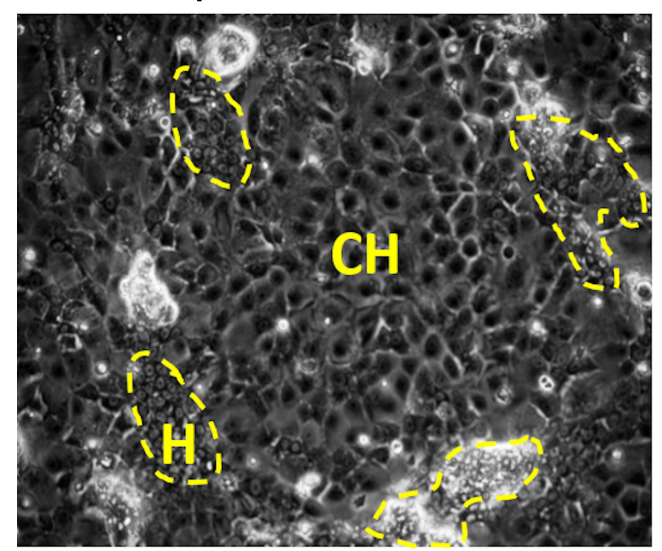

HepaRG + dobutamine

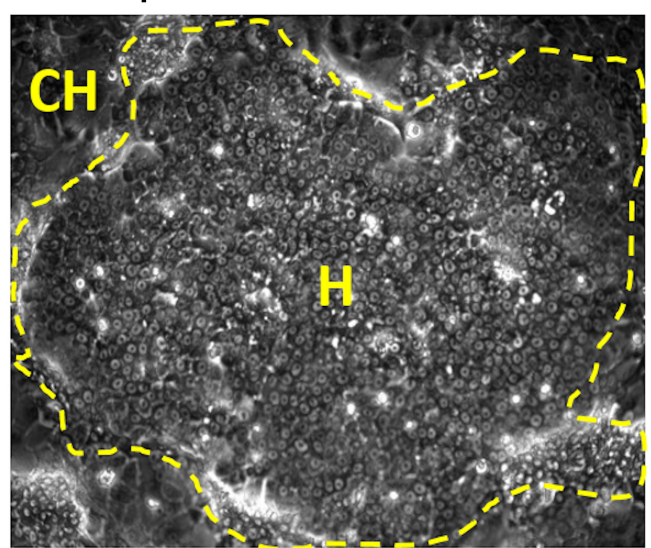

C
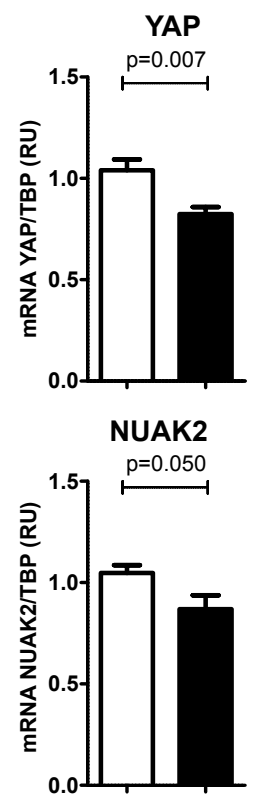
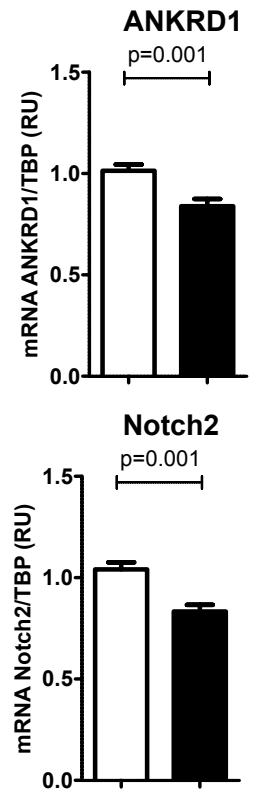

HepaRG treated with vehicle
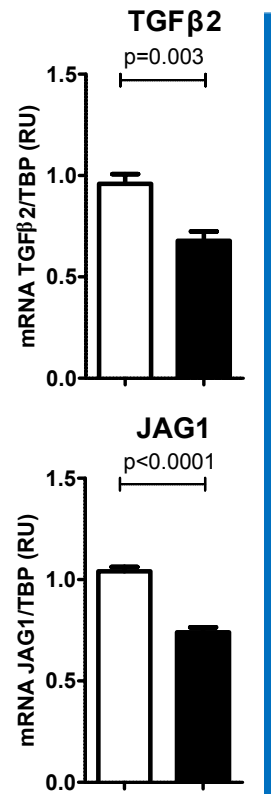

D Hepatocyte markers E Billiary markers
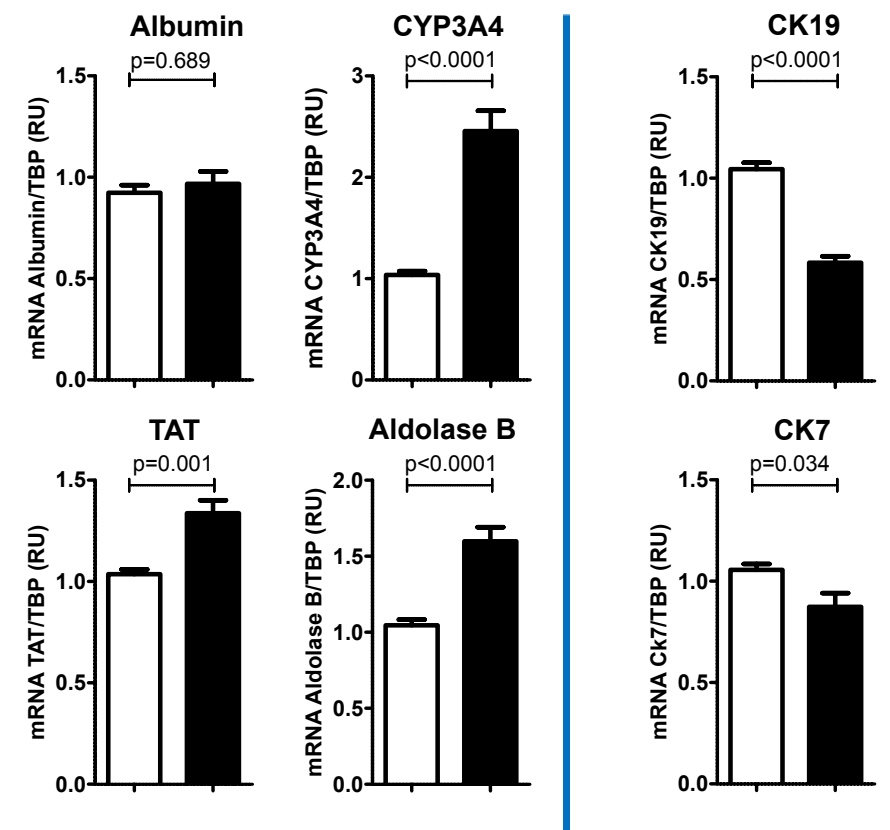

HepaRG treated with dobutamine 
Figure 6

AAlcoholic hepatitis Primary hepatocytes

Dobutamine
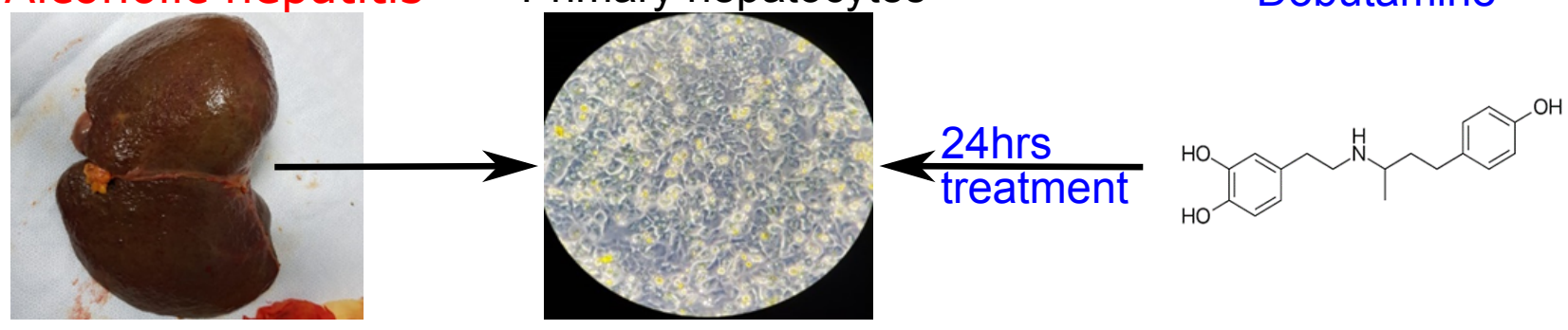

B YAP and target genes

C Hepatocyte markers

D Biliary markers
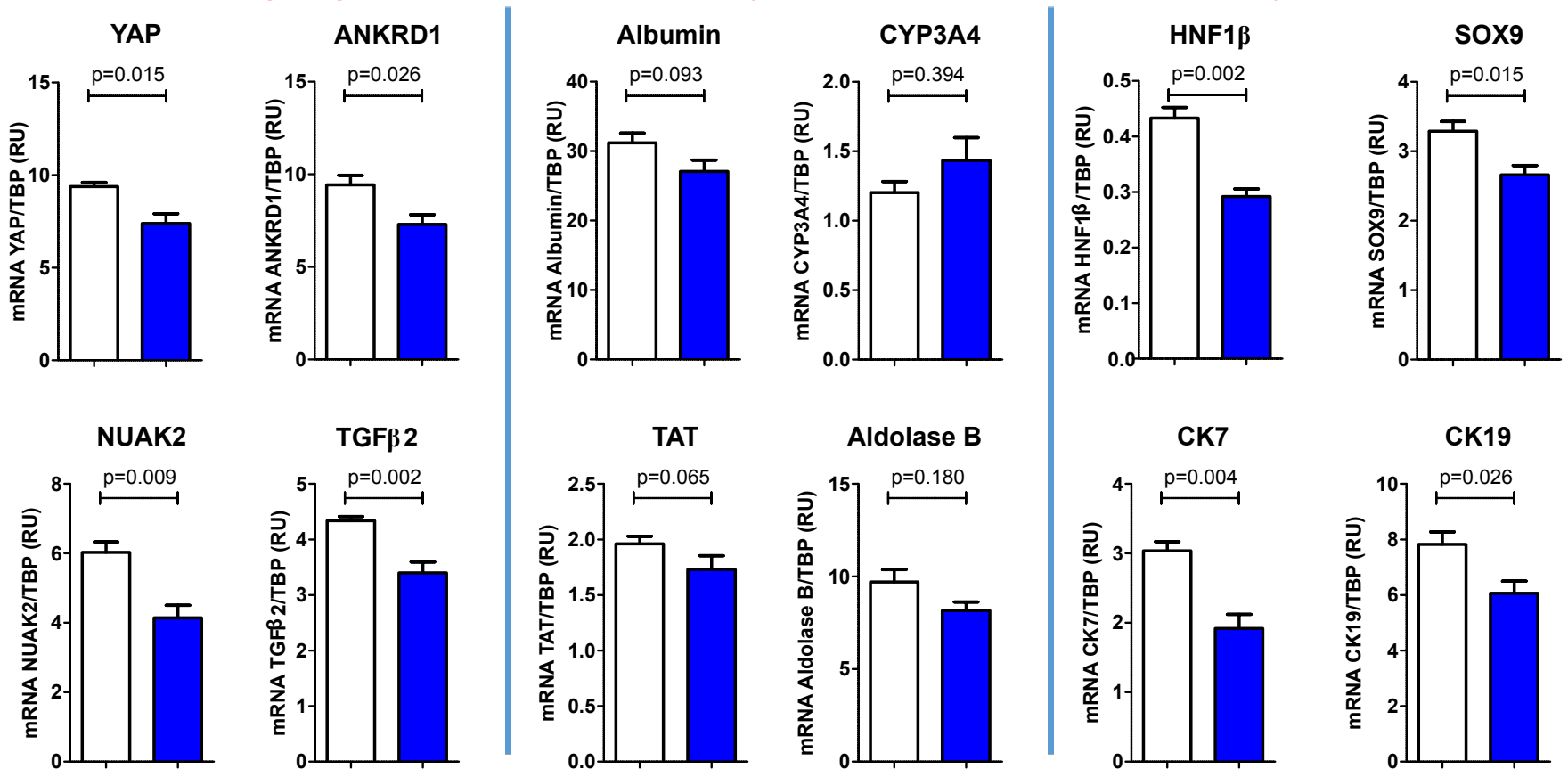

Aldolase B
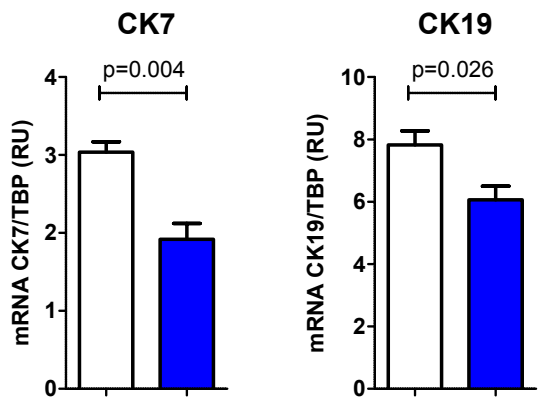

$\mathrm{AH}$ hepatocytes treated with vehicle

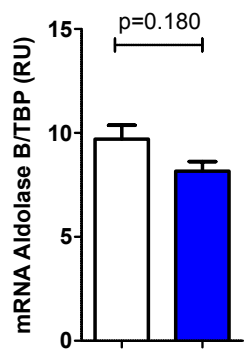

AH hepatocytes treated with dobutamine 
Figure 7

A
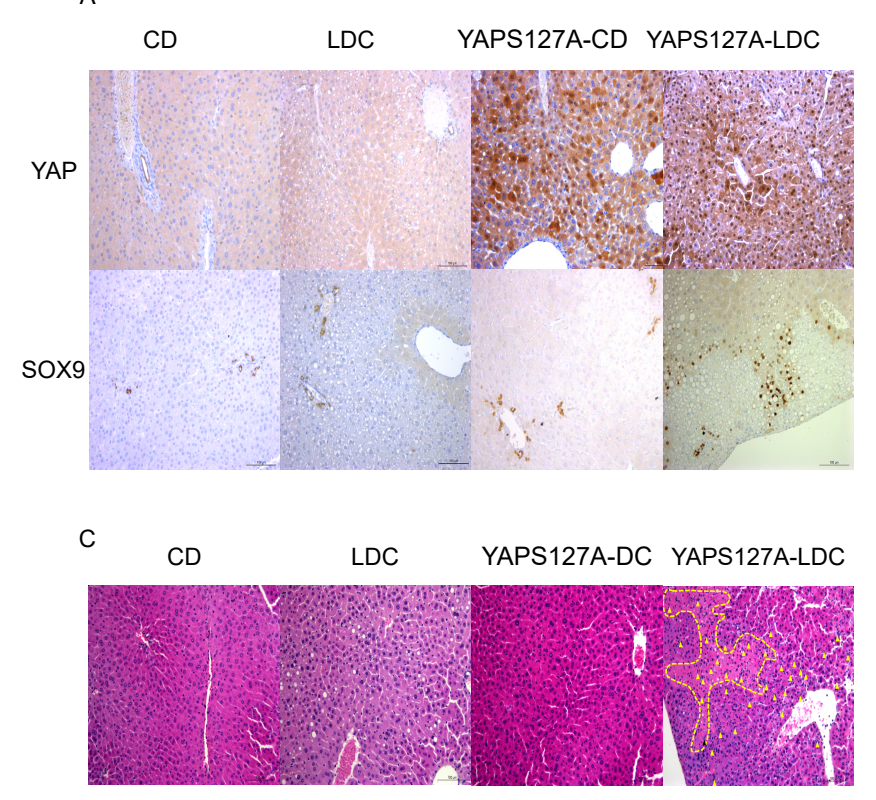

E
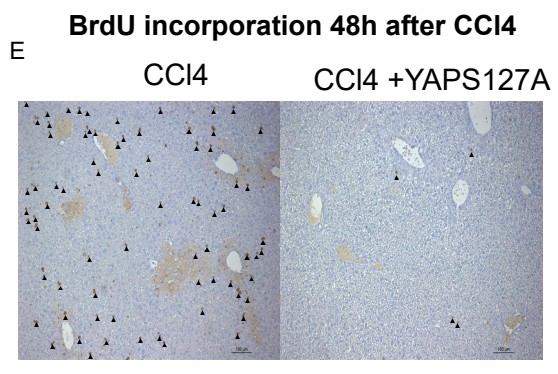

B
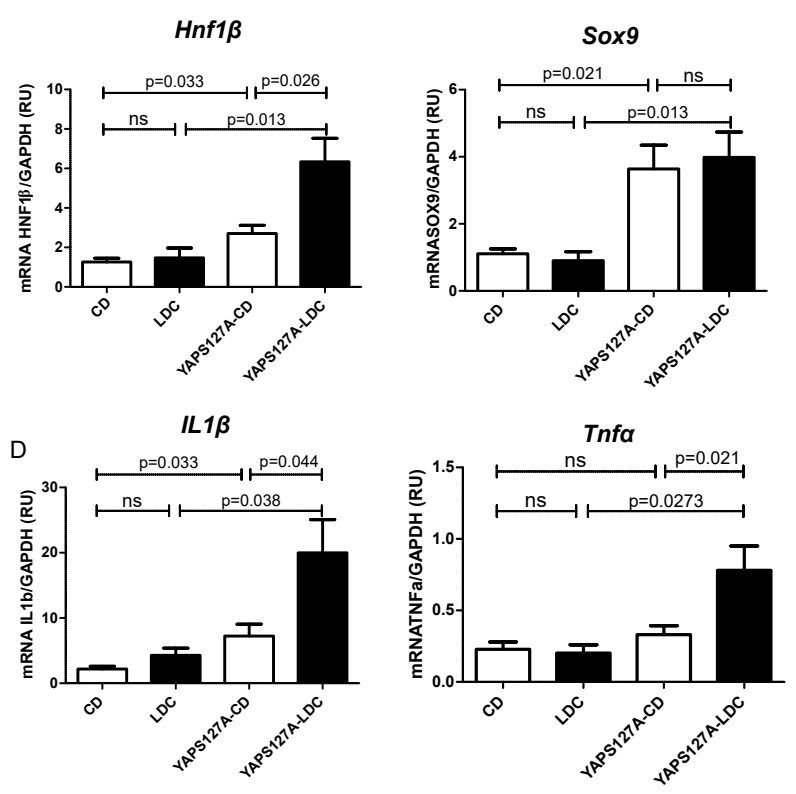

G
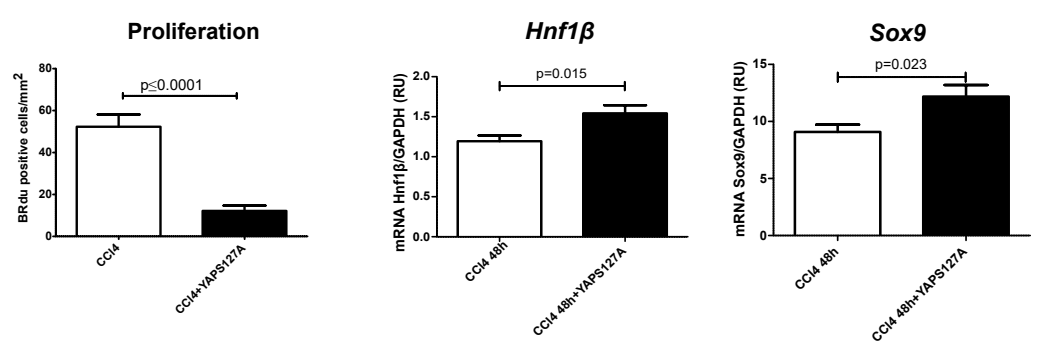
Graphical abstract

Alcoholic hepatitis

Alcoholic cirrhosis

Control liver
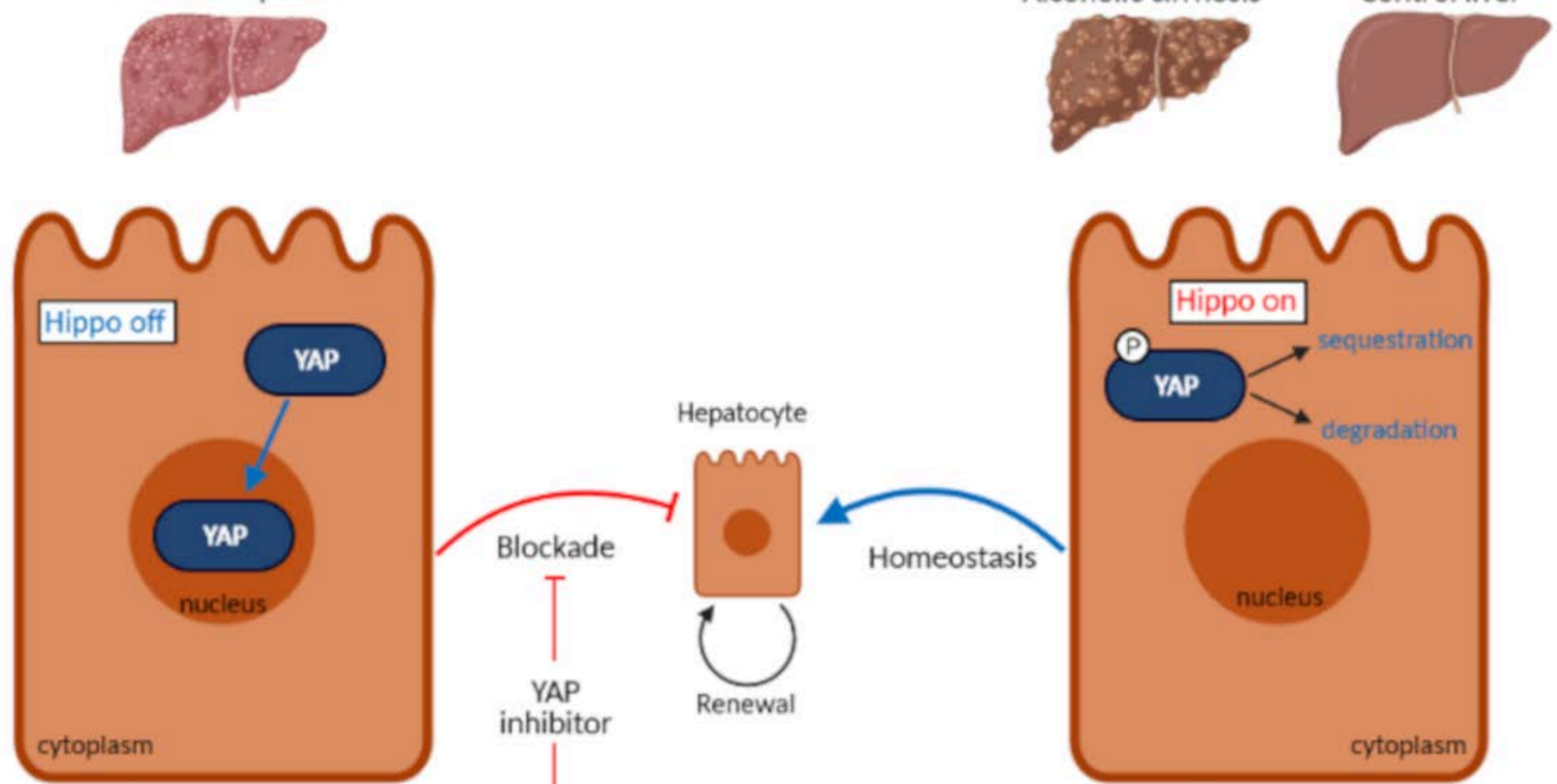

Hepatocyte

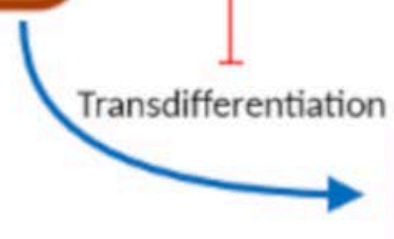

\section{Hepatocyte}
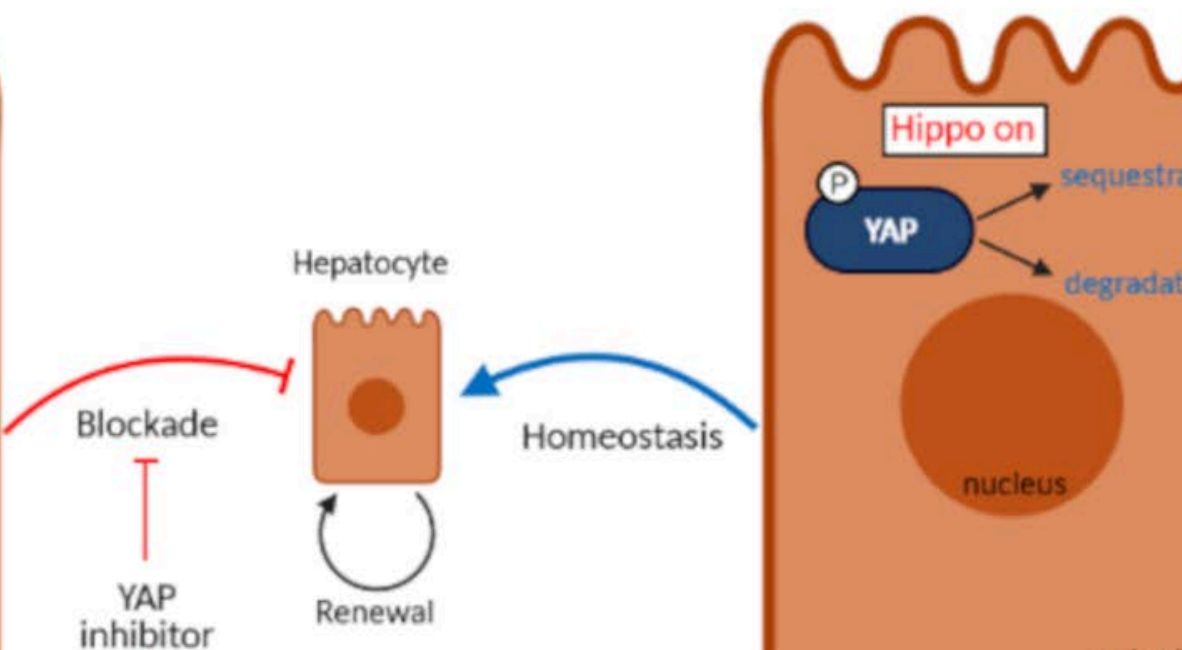

Homeostasis

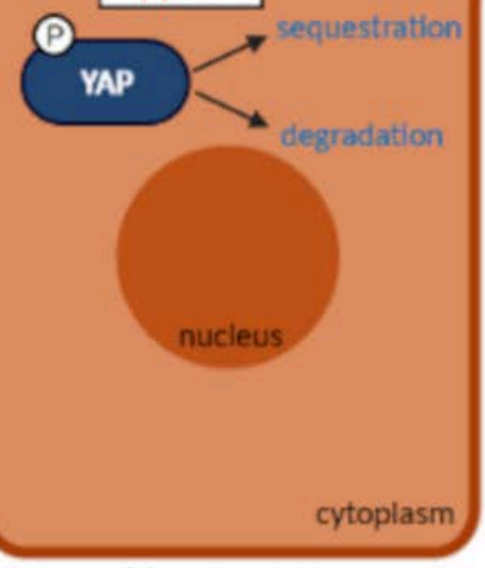

Hepatocyte

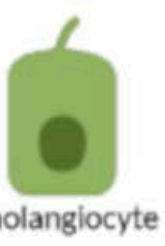

